Liang, K, Zhao, L, Zhao, X, Wang, Y and Ou, S

Joint Resource Allocation and Coordinated Computation Offloading for Fog Radio Access Networks.

Liang, K, Zhao, L, Zhao, X, Wang, Y and Ou, S (2017) Joint Resource Allocation and Coordinated Computation Offloading for Fog Radio Access Networks. China Communications, 13 (Supplement2). pp. 131-139.

doi: 10.1109/CC.2016.7833467

This version is available: https://radar.brookes.ac.uk/radar/items/c17485d3-21fa-4410-9602-27bfdfae1af5/1/

Available on RADAR: February 2017

Copyright (C) and Moral Rights are retained by the author(s) and/ or other copyright owners. A copy can be downloaded for personal non-commercial research or study, without prior permission or charge. This item cannot be reproduced or quoted extensively from without first obtaining permission in writing from the copyright holder(s). The content must not be changed in any way or sold commercially in any format or medium without the formal permission of the copyright holders.

This document is the published version of the journal article. 


\title{
Joint Resource Allocation and Coordinated Com- putation Offloading for Fog Radio Access Networks
}

\author{
Kai Liang ${ }^{1}$, Liqiang $\mathrm{Zhao}^{1}$, Xiaohui Zhao ${ }^{1}$, Yong Wang ${ }^{1}$, Shumao Ou ${ }^{2}$ \\ ${ }^{1}$ State Key Laboratory of Integrated Service Networks, Xidian University, 2 Taibai South Road, Xi'an, Shaanxi, 710071, China \\ ${ }^{2}$ Department of Computing and Communication Technologies, Oxford Brookes University, Wheatley, Oxfordshire, OX33 1HX, UK
}

\begin{abstract}
The cloud radio access network (C-RAN) and the fog computing have been recently proposed to tackle the dramatically increasing traffic demands and to provide better quality of service (QoS) to user equipment (UE). Considering the better computation capability of the cloud RAN (10 times larger than that of the fog RAN) and the lower transmission delay of the fog computing, we propose a joint resource allocation and coordinated computation offloading algorithm for the fog RAN (F-RAN), which takes the advantage of C-RAN and fog computing. Specifically, the F-RAN splits a computation task into the fog computing part and the cloud computing part. Based on the constraints of maximum transmission delay tolerance, fronthaul and backhaul capacity limits, we minimize the energy cost and obtain optimal computational resource allocation for multiple UE, transmission power allocation of each UE and the event splitting factor. Numerical results have been proposed with the comparison of existing methods.
\end{abstract}

Keyword: fog RAN, C-RAN, computation offloading, resource allocation

\section{INTRODUCTION}

Today, ubiquitous mobile devices squeezing into the current network have dramatically changed people's everyday life and posed challenges on network management and data processing. This imposes an explosive growth of intensive computation and the energy consumption on these mobile devices, because mobile services (for communication, entertainment, remote education, face recognition and social networking) normally consume higher energy and require high computation capabilities. Unfortunately, mobile devices normally suffer from the limited battery lifetime and lowcomputational capability, which cannot meet the higher energy demands of mobile applications and run such high-computational applications. Besides, today's centralized networks adopted cloud data center to provide services (i.e., data processing and management), have the problems of low network resource utilization and long service delays, especially for the frequent-services of the edge users.

Computation offloading is a promising approach for mobile devices to offload their most energy-consuming tasks to more resourceful servers remotely or nearby, thereby mitigating the bottleneck of battery life and the computing resources. Cloud computing using cloud data centers for centralized provisioning of data processing, management, and services enables computation offloading. Meanwhile, cloud radio 
access network (C-RAN), has been presented and soon received a large amount of attention in both academic and industry [1], where the centralized base band unit (BBU) takes charge in the baseband processing and connects the densely deployed remote radio heads (RRHs) through fronthaul links. Many works have the computation offloading in C-RAN. In [2], whether computation offloading can save energy was studied. The authors in [3] proposed a wide cross-layer optimization computation offloading strategy for mobile cloud computing. In [4], the authors studied a dynamic offloading algorithm based on Lyapunov optimization, achieving energy saving and meeting the demands of application execution time. [5] investigates collaborative task execution between a mobile device and a cloud clone for mobile applications under a stochastic wireless channel. [6] investigates the problem of mobilityassisted opportunistic computation offloading by exploiting the contact patterns regulated by these devices' mobility.

However, cloud computing and C-RANs are facing a fairly low network resource utilization and long delays. In practice, the fronthaul of C-RAN is often capacity and timedelay constrained, which has a significant decrease on spectral efficiency (SE) and energy efficiency (EE) gains. Fog computing [7] has emerged to move a majority of computation tasks and services from the cloud to the edge of networks, relieving the overloaded cloud data centers. Fog RAN (F-RAN) [8] takes the advantage of fog computing and C-RAN and overcomes the drawbacks of C-RANs with the fronthaul constraints, which can be classified into distributed and centralized F-RAN. In the distributed F-RAN [9-11], the BBU drifts some functionalities (such as data processing, storage, and resource management) to RRUs and even user equipment. In contrast, the centralized F-RAN [12-15] utilizes the emerging concept of software defined networking (SDN) and network virtualization, which facilitates logically centralized control plan and easier management as well as resource sharing. In centralized F-RANs, fog nodes are of better capabilities than RRUs in distributed F-RANs and provide local services to UE, while RRUs only transmit and receive the radio frequency signals. The fog nodes connect RRUs and the cloud center through fronthaul links and backhaul links, respectively. F-RANs has apparent advantages, including the realtime collaboration radio signal processing (CRSP) and flexible cooperative radio resource management (CRRM) at the edge devices, the rapid and affordable scaling that make F-RANs adaptive to the dynamic traffic and radio environment, and low burden on backhaul links and the cloud center.

F-RANs have been studied in some works. A network architecture of distributed F-RANs was discussed in [9], which enables local data processing, coordinated resource management and distributed storage. In [10], the optimal design of F-RANs was studied, which jointly considers edge caching, fronthaul capacity, and radio resources. The computational and radio resource problems were discussed in [11]. In [12], the definition of fog computing and the corresponding technologies including software defined network and network virtualization were discussed. In [13], a logical hierarchy of mobile F-RANs was provided, where the fog node plays a role of intermediate mode between the UE and the could center. A SDN-based F-RAN for vehicular Ad-hoc networks was proposed in [14]. In [15], we have proposed a software defined and virtualized radio access network with fog computing, where a hierarchical control plane network facilitates fog computing and can be viewed as a centralized F-RAN.

Cloud computing has higher computing capability but suffers from the large transmission delay, while fog computing with lower computing capability can provide offloading to the nearby users. Since the research on fog computing and F-RANs is in the infant stage, there are challenges in operation strategies including how to make a computation offloading decision and achieve cooperation between the fog and cloud computing.

Based on our previous work on the SDNbased F-RAN architecture in [15], this paper 
studies the resource allocation policies and the coordinated offloading method in the centralized F-RANs. We formulate the problem as minimizing the energy consumption of offloading all the user equipment (UE)'s computation tasks with satisfying the per-UE delay tolerance, the fronthaul and backhaul capacity constraints, and the available resource constraints. The main contribution of this paper are summarized as follows. First, compared with existing methods for the distributed, we focus on the resource allocation and computation offloading methods for centralized F-RANs. The centralized F-RAN is based on SDN and network virtualization, and thus enables easy management (such as the cooperation between fog nodes and the cloud center) and resource sharing. The fog nodes have higher computing capability and larger storage than RRUs in distributed F-RANs. Second, we provide a coordinated offloading method, which splits the UE's task into cloud computing part and the fog computing part. The fog computing part of the task is executed in the fog, and the remain part is executed in the cloud. Third, we also joint optimization the UE's transmission power, the computing resources in the fog and the cloud, and the cooperation factor (the ratio of the fog computing part to the whole offloading task), to reduce the energy consumption of
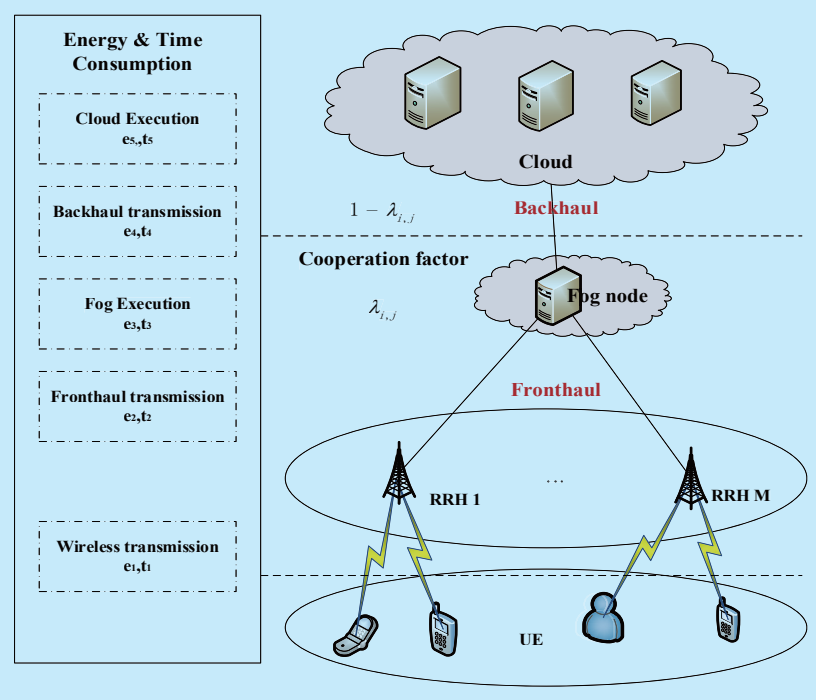

Fig. 1 System model. computation offloading.

The rest of this paper is organized as follows. In Section II, we introduce the system model of the centralized F-RAN. In Section III, we formulate the problem and provide the joint resource allocation and coordinated offloading algorithm. In Section IV, numerical results are provided, and we conclude this paper in Section V.

\section{SYSTEM MODEL}

In this section, we will present the network architecture, task model, and the execution model of the centralized F-RANs.

\subsection{Network architecture and task model}

We consider a F-RAN consisting of a fog node with the capability of storage and computing, $M$ radio remote units (RRUs) with each serving $J$ UEs. Each RRU and UE are equipped with $N_{t}>$ 1 and signal antennas, respectively. Assume that radio frequency (RF) signals are transmitted on a single frequency band. The RRU transmits data to the fog node via the fronthaul links, and the fog node transmits data to the cloud through backhaul links.

Assume that the UE's computation task should be executed on the fog node or the cloud. Denote computation task of the $\mathrm{j}$-th UE served by the i-th RRU as $U_{i, j}=\left\{B_{i, j}, I_{i, j}\right\}$. $B_{i, j}$ stands for the size of command (the input data for executing the task), which should be transmitted from the UE to the fog and the cloud. $I_{i, j}$ represents the total calculation quantity of finishing the computation task. We assume that all the computation tasks of each UE are separable, enabling the fog and the cloud to cooperatively execute the UEs' tasks. For example, the execution of the computation task can be split into two parts. One part will be executed in the fog (accounts for $0 \leq \lambda \leq 1$ ), and the remain part will be executed in the cloud. We denote $\lambda$ as the cooperation factor. Based on the collected network status, the fog node allocates the available resources, and sets up the offloading strategies. 


\subsection{Execution model}

In this paper, we emphasize the cooperative computation offloading between the fog and the cloud. Specifically, each task can be offloaded to the fog and the cloud. In this configuration, we consider six stages involved in the $\mathrm{UE}_{\mathrm{i}, \mathrm{j}}$ 's offloading task.

There are four data transmission stages in this paper, including wireless transmission, wired transmission (from RRU to the fog node, from the fog node to the cloud), and the computation results feedback stage. Note that since the size of results is normally small compared with the input data, we ignore energy and time consumption of the feedback stage.

In wireless transmission stage, the UE transmits the input data of executing task to its serving RRU. We consider a time slotted block fading channel. Assume that perfect channel state information (CSI) is available at the fog node. Zero-forcing [16] signal detecting together with uplink coordinated multipoint (CoMP) technique is used to eliminate multi-user interference and the inter-cell interference [17]. The data rate of the j-th UE in the i-th cell is given by:

$$
R_{i, j}=W \log _{2}\left(1+\frac{P_{i, j}\left|\mathbf{h}_{i, j} \mathbf{v}_{i, j}^{\mathrm{CoMP}-\mathrm{ZF}}\right|^{2}}{W \sigma^{2}}\right)
$$

where $W \mathrm{MHz}$ denotes the bandwidth of wireless channel; $\mathbf{h}_{i, j}$ denotes the wireless channel between $\mathrm{UE}_{\mathrm{i}, \mathrm{j}}$ and $\mathrm{RRU}_{\mathrm{i}}$, suffering a joint path loss and multipath fading; $\mathbf{v}_{i, j}^{\text {CoMP-ZF }}$ denotes CoMP-ZF signal detecting vector at the $\mathrm{RRU}_{\mathrm{i}}$ for $\mathrm{j}$-th UE; $\sigma^{2}$ represents the power of complex additive white Gaussian noise (AWGN); $P_{i, j}$ is the transmission power of the $\mathrm{UE}_{\mathrm{i}, \mathrm{j}}$. During the wireless transmission phase, the transmission time and energy consumption are $t_{1, i, j}=\frac{B_{i, j}}{R_{i, j}}$ and $e_{1, i, j}=t_{1, i, j} P_{i, j}$.

Denote transmission capacity of the fronthaul and backhaul links as $C_{F H}$ and $C_{B H}$ Mbps. With the consideration of user fairness, the transmission capacities for each UE in the fronthaul and backhaul links are upper bounded by $\mathrm{d}$ and c Mbps. Denote the power consumption of RRU and the fog node processing and transmitting input data to the fog and the cloud as $P_{R F}$ and $P_{F C}$. Therefore, the time and energy consumption during the input data transmission in the fronthaul links are $t_{2, i, j}=\frac{B_{i, j}}{c}$, and $e_{2, i, j}=t_{2, i, j} P_{R F}$, and the consumption in the backhaul transmission are $t_{4, i, j}=\frac{B_{i, j}}{d}$, and $e_{4, i, j}=t_{4, i, j} P_{F C}$.

Denote the computation capability of the fog and the cloud as $S_{f}$ and $S_{c}$ million instructions per second (MIPS). The computing resources allocated for the $\mathrm{UE}_{\mathrm{i}, \mathrm{j}}$ in the fog and in the cloud are denoted as $S_{f, i, j}$ and $S_{c, i, j}$. The execution time and the energy consumption of the fog are $t_{3, i, j}=\frac{I_{i, j}}{S_{f, i, j}}$, and $e_{3, i, j}=t_{3, i, j} P_{F}$, and those of the cloud are $t_{5, i, j}=\frac{I_{i, j}}{S_{c, i, j}}$, and $e_{5, i, j}=t_{5, i, j} P_{C}$, where $P_{F}$ and $P_{C}$ represent the power consumption of executing task in the fog and the cloud, respectively.

\section{Problem Formulation and Resource Allocation Algorithm}

In this section, we will formulate the problem and give the resource allocation algorithm to obtain the optimal solutions.

\subsection{Problem formulation}

In this paper, we focus on the energy consumption that all the UEs offload their tasks to the fog and the cloud. Therefore, the optimal problem can be formulated as minimizing the sum of energy consumption with satisfying the delay tolerance of each task, and the available resource constraints, which is given by (2)

$$
\begin{gathered}
\min _{\substack{P_{i, j}, \lambda_{i, j}, S_{f, j}, S_{c, i, j}}} \sum_{i=1}^{M} \sum_{j=1}^{J}\left(e_{1, i, j}+e_{2, i, j}+\lambda_{i, j} e_{3, i, j}+\left(1-\lambda_{i, j}\right)\left(e_{4, i, j}+e_{5, i, j}\right)\right) \\
\text { s.t. } \mathrm{C} 1: t_{1, i, j}+t_{2, i, j}+\lambda_{i, j} t_{3, i, j}+\left(1-\lambda_{i, j}\right)\left(t_{4, i, j}+t_{5, i, j}\right) \leq T_{i, j} \\
\mathrm{C} 2: R_{i, j} \leq \min (c, d) \quad \mathrm{C} 3: \sum_{i=1}^{M} \sum_{j=1}^{J} R_{i, j} \leq \min \left(C_{F H}, C_{B H}\right) \\
\mathrm{C} 4: \sum_{i=1}^{M} \sum_{j=1}^{J} S_{f, i, j} \leq S_{f} \quad \mathrm{C} 5: \sum_{i=1}^{M} \sum_{j=1}^{J} S_{c, i, j} \leq S_{c} \\
\mathrm{C} 6: 0 \leq P_{i, j} \leq P_{U E}, 0 \leq \lambda_{i, j}<1, S_{f, i, j} \geq 0, S_{c, i, j} \geq 0 \\
i=1, \cdots, M, j=1, \cdots, J
\end{gathered}
$$

where the objective function represents the energy consumption of offloading all the UEs' 
computation tasks and $P_{U E}$ is the maximum transmission power of each UE. C1 indicates that the time consumption on offloading a task of $\mathrm{UE}_{\mathrm{i}, \mathrm{j}}$ should be no more than the delay tolerance $T_{i, j}$. C2 denotes the transmission rate of each UE should be less than upper bound of per-UE allocated fronthaul and backhaul capacity. C3 denotes that the sum rate of all the UE should be limited by the fronthaul and backhaul capacity. C4 and C5 indicate the allocated computing resource for UEs cannot exceed the maximum available computation resources in the fog and the cloud.

Problem (2) is a non-convex problem, due to the products of variables in the objective function, the reverse convex constraints $\mathrm{C} 3$ as well as $e_{1, i, j}$. Therefore, it is difficult to solve problem (2) and to obtain the optimal solution. In the following sub-section, we will reformulate problem (2) and get an approximate solution.

\subsection{Solution}

In the objective function, $e_{1, i, j}$ is concave and other parts of the objective function are constant or convex. Note that

where

$$
e_{1, i, j} \leq \tilde{e}_{1, i, j}=\frac{\tilde{P}_{i, j} B_{i, j}}{R_{i, j}}
$$

$$
\tilde{P}_{i, j}=\min \left(\frac{\left(2^{\frac{\min (c, d)}{W}}-1\right) \cdot W \sigma^{2}}{\left|\mathbf{h}_{i, j} \mathbf{v}_{i, j}^{\text {CoMP-ZF }}\right|^{2}}, P_{U E}\right)
$$

We can use $\tilde{e}_{1, i, j}$ to approximate $e_{1, i, j}$ in the objective function. Since $\tilde{e}_{1, i, j}$ is convex, the approximated objective function is convex.

The constraint C3 is reverse convex [18], making it difficult to solve problem (2). However, since constraint C2 limits the upper transmission rate of each UE, we can adopt access control [19] in the network to ensure the number of UE will not exceed $\min \left(\frac{C_{F H}}{c}, \frac{C_{B H}}{d}\right)$. This practice enables us to ignore the constraint $\mathrm{C} 3$, and therefore (2) is relaxed as follows:

$$
\begin{aligned}
\min _{\substack{P_{i, j}, \lambda_{i, j}, S_{f, i, j}, S_{c, i, j}}} & \sum_{i=1}^{M} \sum_{j=1}^{J}\left(\tilde{e}_{1, i, j}+e_{2, i, j}+\lambda_{i, j} e_{3, i, j}\right. \\
& \left.+\left(1-\lambda_{i, j}\right)\left(e_{4, i, j}+e_{5, i, j}\right)\right) \\
\text { s.t. } & \mathrm{C} 1, \mathrm{C} 2, \mathrm{C} 4, \mathrm{C} 5, \mathrm{C} 6
\end{aligned}
$$

By fixing the cooperation the factor , problem (5) is a convex problem, and can be solved by conventional convex optimization methods. In the next sub-section, we will provide an alternating algorithm to solve problem (5).

Algorithm 1: Joint resource allocation and coordinated offloading algorithm

Input: $\mathbf{h}_{i, j}, P_{R}, P_{B F}, P_{U E}, P_{F}, P_{C}, S_{f}, S_{c}, B_{i, j}, I_{i, j}$ Output:

$P_{i, j}, \lambda_{i, j}, S_{f, i, j}, S_{c, i, j}$

1. Initialize the cooperative factor $\lambda_{i, j}^{0} \in[0,1]$. Set $\mathrm{k}=1$.

\section{Loop}

3. Update $P_{i, j}^{k}, S_{f, i, j}^{k}, S_{c, i, j}^{k}$, by solving (5) with fixed $\lambda_{i, j}^{k-1}, \forall i, j$.

4. Update $\lambda_{i, j}^{k}$, by solving (5) with fixed $P_{i, j}^{k}, S_{f, i, j}^{k}, S_{c, i, j}^{k}, \forall i, j$

5. If certain stopping criterion is satisfied $\lambda_{i, j}^{*}=\lambda_{i, j}^{k}, P_{i, j}^{*}=P_{i, j}^{k}$,

6. $S_{f, i, j}^{*}=S_{f, i, j}^{k}, S_{c, i, j}^{*}=S_{c, i, j}^{k}$

7. Break.

8. End if

9. $\mathrm{k}=\mathrm{k}+1$.

10. End loop

\subsection{Resource allocation algorithm}

In this sub-section, we propose a coordinated offloading algorithm to solve problem (5) by using an alternating convex optimization method [20]. By updating power and computing resource, and the cooperation factors alternatively, we can get the optimal solutions until the convergence condition is satisfied. The algorithm is summarized in Algorithm 1.

\section{Numerical Results}

To testify the performance of the proposed algorithm, numerical results are presented in this section. Throughout the simulations, the following settings are used unless stated otherwise. Assume that there are two RRUs $\left(N_{t}\right.$ $=8$ ) with each served 2 UEs. The maximum transmission power of UE $P_{U E}=0.1 \mathrm{~W}$ and the power of noise is $\sigma^{2}=10^{-8} \mathrm{~W}$. The distance between each UE to its serving RRH is 50 
meters, and the path loss exponent is 2 . The wireless channel follows Rayleigh fading, with zero mean and unit variance. The wireless bandwidth is $10 \mathrm{MHz}$. The fronthaul and backhaul capacity are $C_{F H}=12 \mathrm{Mbps}$ and $C_{B H}$ $=100 \mathrm{Mbps}$ [21]. The power consumptions of transmitting input data in the fronthaul and backhaul are $1 \mathrm{~W}$ and $10 \mathrm{~W}$. The size of perUE's input data $B_{i, j}=10$ Mbits. The quantity of per-UE's computation $I_{i, j}=100$ million instructions (MIs). The computing capabilities of the fog and the cloud are 100 MIPS and 1000 MIPS, with the power cost of $1 \mathrm{~W}$ and $4 \mathrm{~W}$, respectively.

Fig. 2 shows the average iteration numbers of the proposed algorithm under the various size of input data. We can find that the proposed algorithm can always obtain the solutions within 2 iterations and shows a fast convergence property.

Fig. 3 shows the energy consumption versus various sizes of input data. We compare our proposed algorithm with the traditional cloud computing. Generally, the energy consumption increases with the increasing size of input data, which spends more energy on the input data transmission. The proposed algorithm shows lower energy consumption than that of cloud computing because a portion of the UE's task can be executed in the fog node and this saves the energy cost of input data transmission between the fog node to the cloud. We can observe that when the size of input data ranges from 2.5 to 17.5 Mbits, the energy consumptions of our proposed algorithm under the different backhaul capacities remain the same, because the fog node provides offloading services without energy consumption in fronthaul transmission in this case. In contrast, when the input data size becomes larger, the cloud undertakes a part of task execution to ensure the delay tolerance. Therefore, the energy consumption in the larger backhaul capacity is lower than that in small backhaul capacity. The proposed algorithm allocates computation tasks on the fog node and cloud server based on the parameter $\lambda_{i, j}$, which implies proportion of the task executed on the fog node

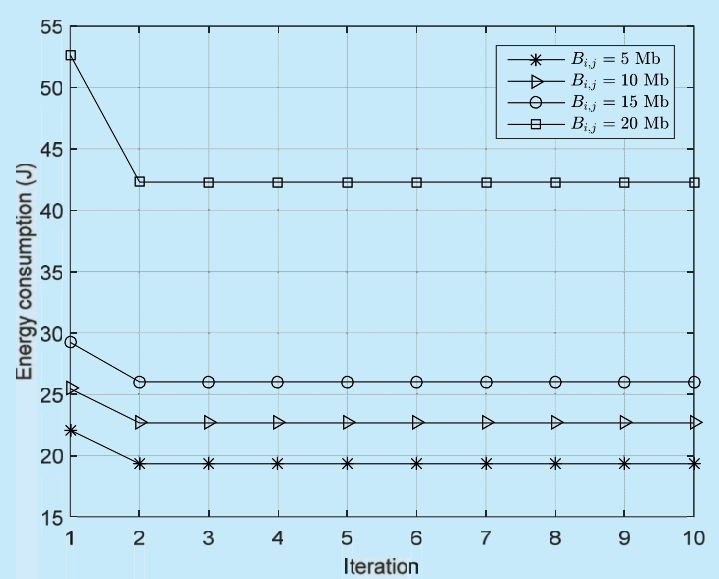

Fig.2 Average iteration numbers

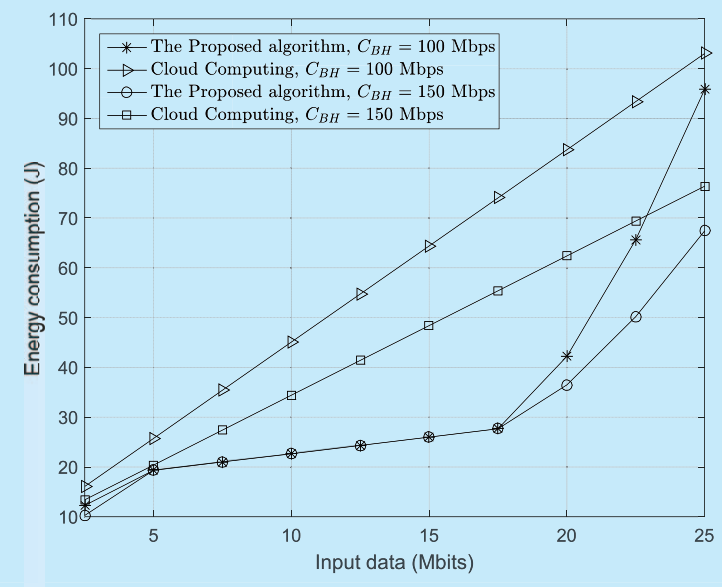

Fig.3 Energy consumption versus input data

and cloud server. For this reason, the energy consumption of the proposed algorithm is upper bounded by that of cloud computing.

Fig. 4 shows the energy consumption versus the quantity of computation, namely the number of instructions. The energy consumptions of the four curves show an upward trend, with the growing number of instructions. The proposed algorithm shows a better performance than the cloud computing. It is worth noting that in the case of larger number of instructions, i.e., more than $400 \mathrm{MIs}$, the proposed algorithm remains the same energy consumption with cloud 


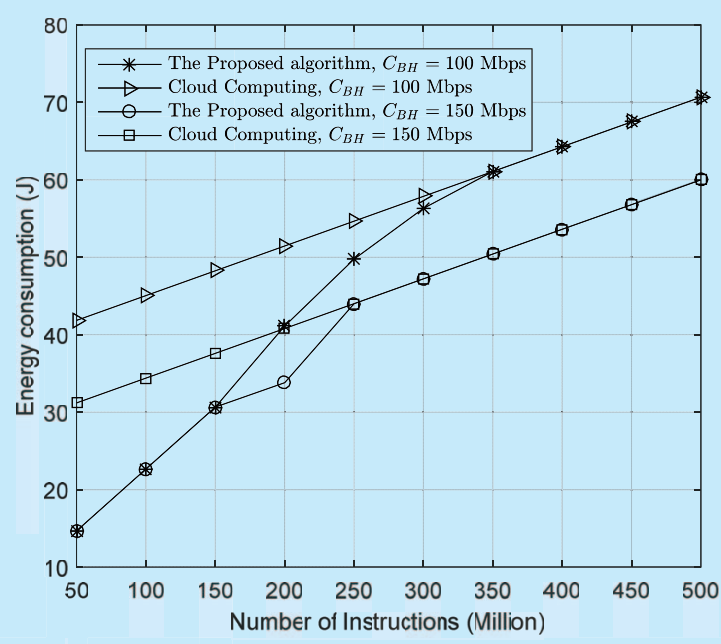

Fig.4 Energy consumption versus number of instructions

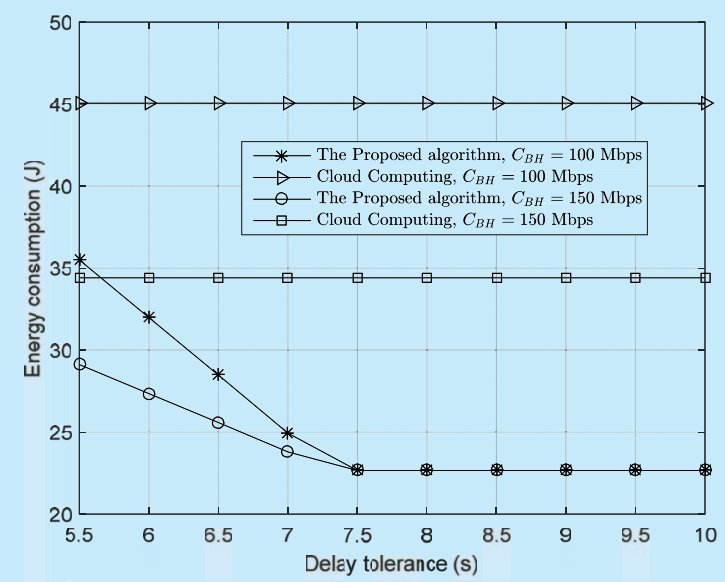

Fig.5 Energy consumption versus delay tolerance

computing. This is because in this case, all the instructions should be executed in the cloud to guarantee the delay tolerance.

Fig.5 shows the energy consumption versus the different delay tolerances. The energy consumption of the cloud computing remains constant with the increasing delay tolerances, because cloud computing cannot reduce the transmission delay. In contrast, the proposed algorithm first decreases and then remain the constant with the increasing delay tolerances. Since the transmission delay between the fog to the cloud is less than the execution delay in the fog, parts of the instructions are executed in the cloud when the delay tolerance is small. However, when the delay tolerance goes large, a majority of the UE's task will executed in the fog for the sake of reducing energy consumption. This indicates that fog computing is suitable for small quantity of computation tasks.

\section{CONCLUSION}

In this paper, we study a joint resource allocation and coordinated offloading method for the F-RANs. With the assumption of separable offloading tasks, the UE's task can be cooperatively executed in the fog and the cloud. Specifically, a portion of the task is executed in the fog, and the remain part is executed in the cloud. We formulate the problem as minimizing the energy consumption of offloading all the UEs' tasks, with satisfying per-UE delay tolerance, per-UE transmission power constraint, fronthaul and backhaul capacity constraints, and the computing resource constraints. The numerical results show that the proposed algorithm can guarantee the delay tolerance while reduce the energy consumption, compared with the traditional cloud computing.

\section{ACKNOWLEDGEMENT}

This work was supported in part by National Natural Science Foundation of China (No. 61372070), Natural Science Basic Research Plan in Shaanxi Province of China (No. 2015JM6324), Ningbo Natural Science Foundation (2015A610117), Hong Kong, Macao and Taiwan Science \& Technology Cooperation Program of China (No. 2015DFT10160), EU FP7 Project MONICA (No. PIRSES-GA-2011-295222), the 111 Project (No. B08038).

\section{References}

[1] F. Bonomi, R. Milito, J. Zhu, and S. Addepalli, "Fog computing and itsrole in the Internet of Things", In Workshop on Mobile Cloud Computing, 
MCC'12, Helsinki, Finland, pp. 13-16, Aug. 2012.

[2] K. Kumar and Y. H. Lu, "Cloud computing for mobile users: Can offloading computation save energy?", Computer, vol. 43, no. 4, pp. 51-56, Apr. 2010.

[3] S. Barbarossa, S. Sardellitti, and P. Di Lorenzo, "Computation offloading for mobile cloud computing based on wide cross-layer optimization", in Proc. Future Netw. Mobile Summit (FuNeMS'13), Lisboa, Portugal, pp. 1-10, Jul.3-5, 2013.

[4] D. Huang, P. Wang, and D. Niyato, "A dynamic offloading algorithm for mobile computing", IEEE Trans. Wireless Commun., vol. 11, no. 6, pp. 1991-1995, Apr. 2012.

[5] W. Zhang, Y. Wen, and D. Oliver, "Collaborative task execution in mobile cloud computing under a stochastic wireless channel", IEEE Trans. Wireless Commun, vol. 14, no. 1, pp. 81-93, January. 2015.

[6] C. Wang, Y. Li and D. Jin, "Mobility-assisted opportunistic computation offloading", IEEE COMMUNICATIONS LETTERS, vol. 18, no. 10, pp. 1779-1782, October. 2014.

[7] S. Sarkary, S. Chatterjee, and S. Misra, "Assessment of the Suitability of Fog Computing in the Context of Internet of Things," IEEE Trans. Cloud Computing, DOI: 10.1109/TCC.2015.2485206, 2015.

[8] H. Xiang, M. Peng, Y. Cheng and H-H Chen, "Joint mode selection and ressource allocation for downlink fog radio access networks supported D2D", 2015 QSHINE, pp. 117-182, 2015.

[9] M. Peng, et al. "Fog-computing-based radio access networks: issues and challenges", IEEE Network, vol.30, no. 4 pp. 46-53, 2015.

[10] R. Tandon and O. Simeone. "Harnessing cloud and edge synergies: toward an information theory of fog radio access networks", IEEE Communications Magazine, vol. 54 no.6, pp. 4450, 2016

[11] S. Sardellitti, G. Scutari, and S. Barbarossa, "Joint optimization of radio and computational resources for multicell mobile-edge computing", IEEE Transactions on Signal and Information Processing over Networks, vol. 1, no. 2, pp. 89103, Jun. 2015.

[12] L. M. Vaquero, L. Rodero-Merino. "Finding your way in the fog: Towards a comprehensive definition of fog computing", ACM SIGCOMM Computer Communication Review, vol. 44, no. 5, pp. 27-32, 2014.
[13] K. Hong, et al. "Mobile fog: A programming model for large-scale applications on the internet of things. Proceedings of the second ACM SIGCOMM workshop on Mobile cloud computing. ACM, 2013.

[14] N. B. Truong et al. "Software defined networkingbased vehicular Adhoc Network with Fog Computing", 2015 IFIP/IEEE International Symposium on Integrated Network Management (IM), 2015.

[15] Kai Liang, Liqiang Zhao, Xiaoli Chu, and HsiaoHwa Chen, "An Integrated Architecture for Software Defined and Virtualized Radio Access Networks with Fog Computing", IEEE Network, 2016. (In Press). Availabe at http://web.xidian. edu.cn/lqzhao/files/20160923_082655.pdf.

[16] J. Park, G. Lee, Y. Sung and M. Yukawa, "Coordinated Beamforming With Relaxed Zero Forcing: The Sequential Orthogonal Projection Combining Method and Rate Control", IEEE Trans. signal process., vol. 61, no. 12, pp. 3100-3112, 2013.

[17] W Yu, T.Kwon, andC. Shin,MulticellCoordination via Joint Scheduling, Beamforming, and Power Spectrum Adaptation, IEEE Trans. Commun., vol. 12, no. 7, pp. 1-14, 2013

[18] S. Yamada, T. Tanino, M. Inuiguchi and K. Tatsumi, "An inner approximation method for a reverse convex programming problem", IEEE SMC 1999, pp. 521-526, 1999.

[19] Y.Shu, Y. Gu and J. Chen, "Dynamic Authentication with Sensory Information for the Access Control Systems", IEEE Trans. Paral. \& Distributed Systems, vol. 25, no. 2, pp. 427-436, 2014.

[20] Boyd, "EE364b :Convex Optimization II", available at http://stanford.edu/class/ee364b/.

[21] F. Jalali et al., "Fog Computing May Help to Save Energy in Cloud Computing", IEEE Journal on Selected Areas in Communications, vol. 34, no. 5 , pp. 1728 - 1739, 2016.

\section{Biographies}

Kai Liang (the corresponding author, Email: klxdu@ hotmail.com) is now a Ph.D. student in Xi'dian University and majors in communication and information systems. He received his bachelor's degree in communication engineering from Xi'an University of Architecture and Technology, China in 2007. He received the funding from the program of China Scholarships Council to visit the Network Convergence Laboratory, University of Essex, UK, 
Convergence Laboratory, University of Essex, UK, during September2014-September 2015. His current research interests include broadband wireless access, wireless network design, coordinated multipoint-tomultiuser communication systems, and fog computing. Email: klxdu@hotmail.com.

Liqiang Zhao received his Ph.D. degree in information and communications engineering from Xidian University, China, in 2003. He is a full professor with Xidian University. His current research focuses on broadband wireless communications, software defined network and fog computing. He has more than 70 published in authorized academic periodicals. He has hosted/participated a great many research projects, such as the National Natural Science Foundation, 863 program, the EU FP6, FP7 plans, and the Huawei fund. Email: lqzhao@mail.xidian.edu.cn.

Xiaohui Zhao is now studying for her master's degree of communication and information system in Xidian University. She received her bachelor degree of Information Engineering from Xidian University. Her main research interest is fog computing. Email: xxhzhao@hotmail.com.
Yong Wang received the B.S., M.S. and Ph.D. degree from Xidian University, Xi'an, China in 1997, 2002 and 2005 , respectively. He is now a professor of the State Key Laboratory of Integrated Services Networks, Xidian University, China. He is the Vice Chair of IEEE Xian Section Broadcast Technology Society Chapter. His research interests include wireless transmission and nonlinear signal processing. Email: johnnyly@xidian. edu.cn.

Shumao Ou received the M.Sc. (with distinction) and Ph.D. degrees in electronic systems engineering from the University of Essex, Colchester, U.K., in 2004 and 2007, respectively. He is currently a Senior Lecturer in Computer Communications and Networks, Oxford Brookes University, Oxford, U.K. His main research interests include mobile cloud computing, wireless communications, Internet of Things, heterogeneous wireless networks, wireless vehicular communications, and Intelligent Transportation Systems. He manages research project funding from various sources such as, the UK TSP, the European Commission and industries. He has authored or co-authored over 50 technical papers in major international journals and conferences. Email: sou@brookes.ac.uk. 